\title{
Mixed-Valence $\mathrm{CsCu}_{4} \mathrm{Se}_{3}$ : Large Phonon Anharmonicity Driven by the Hierarchy of the Rigid $\left[\left(\mathrm{Cu}^{+}\right)_{4}\left(\mathrm{Se}^{2-}\right)_{2}\right]\left(\mathrm{Se}^{-}\right)$ Double Anti-CaF 2 Layer and the Soft $\mathrm{Cs}^{+}$Sublattice
}

Ni Ma, ${ }^{1}$ Fan Li, ${ }^{2}$ Jian-Gao Li, ${ }^{3}$ Xin Liu, ${ }^{2}$ Dong-Bo Zhang, ${ }^{3}$ Yan-Yan Li, ${ }^{1}$ Ling Chen,${ }^{1}$ 2, *, Li-Ming $W u^{1,2, *}$

${ }^{1}$ Center for Advanced Materials Research, Advanced Institute of Natural Sciences, Beijing Normal University at Zhuhai, Zhuhai 519087, People's Republic of China ${ }^{2}$ Beijing Key Laboratory of Energy Conversion and Storage Materials, College of Chemistry, Beijing Normal University, Beijing 100875, People's Republic of China ${ }^{3}$ College of Nuclear Science and Technology, Beijing Normal University, Beijing 100875, People's Republic of China 


\section{EXPERIMENTAL SECTION}

Synthesis. The reagents of amorphous B powders (99.99\%), $\mathrm{Cs}_{2} \mathrm{CO}_{3}$ powders $(99.99 \%)$, $\mathrm{Cu}$ powders (99.999\%), S powders (99.999\%) and Se powders (99.999\%) were used as purchased from Alfa Aesar. In this study, $\mathrm{CsCu}_{4} \mathrm{Q}_{3}[\mathrm{Q}=\mathrm{S}$ (compound 1), Se (compound 2)] were obtained by Boron-Chalcogen (BC) method: B and S(Se) powder loaded in a silica tube, and the mixture of $\mathrm{Cu}$ and $\mathrm{Cs}_{2} \mathrm{CO}_{3}$ powder was placed in a separated one with same size according to the stoichiometries of $\mathrm{Cs}_{2} \mathrm{CO}_{3}: \mathrm{Cu}: \mathrm{B}$ : $\mathrm{S}(\mathrm{Se})=3: 24: 2: 18$. The assembly was loaded into an evacuated and flame-sealed fused silica tube with larger size and heated to $973 \mathrm{~K}$ over $6 \mathrm{~h}$, held at this temperature for $3 \mathrm{~h}$, then slowly cooled down to room temperature. Afterwards, the raw products were washed with distilled water several times and then dried with ethanol.

Powder and Single-Crystal X-ray Diffractions. The room temperature powder X-ray diffraction measurement (Bruker D8 ADVANCE, $\mathrm{Cu} \mathrm{K} \alpha$ radiation $\lambda=1.5406 \AA$ ) was carried out to verify the chemical composition of target compounds and scanned in the $2 \theta$ range from $10^{\circ}$ to $60^{\circ}$ with step of $0.02^{\circ}$. The intensity data of hand-picked single crystals were collected on Bruker APEX-II CCD diffractometer (Mo K $\alpha \lambda=0.71073$ $\AA$ ) at room temperature. The solutions and refinement for the crystal structure were accomplished by using SHELXTL program package and direct method suggested the P4/mmm space group (No. 123) and offered a rational structure model. The PLATON program confirms the correct space group and structure refinement gives 4.17/8.10\% of final $R_{1} /{ }_{w} R_{2}$ for $1,6.18 / 10.23 \%$ for 2 , respectively. Crystallographic data are listed in Table S1.

X-ray Photoelectron Spectroscopy. XPS instrument (ESCALAB 250Xi, ThermoFisher USA) was used to investigate the chemical composition and valence states of the atoms in $\mathbf{1}, \mathbf{2}$.

Solid-State UV-Vis Spectroscopy. The UV-Vis diffuse reflectance spectra were performed at room temperature with SHIMADZU UV-2600 Spectrometer in the range of 2400-200 nm. 
Thermal Stabilities. Simultaneous thermal analysis (STA) data were measured on a NETZSCH STA 449 F3 analyzer.

Ultrasonic Pulse Echo Measurement. The longitudinal and transverse sound velocities were measured by using a commercial equipment (Ultrasonic Pulser/Receiver Model 5058 PR, Olympus, USA).

Magnetic Property. Magnetic susceptibilities data were collected on a Quantum Design MPMS-3 SQUID magnetometer at 2-300 K under a constant magnetic field of 1000 Oe. And about $150 \mathrm{mg}$ of finely ground sample was loaded into a gelatin capsule. Microscopy Characterization. The high resolution transmission electron microscope (HRTEM) was performed on JEM-ARM200F. The element mapping was identified by Hitachi SU-8010 equipped with an energy-dispersive spectroscopy (EDS) system.

Transport Property Measurement. Finely ground polycrystalline samples were loaded inside to the graphite die $(\Phi=15 \mathrm{~mm})$ and then consolidated by spark plasma sintering (SPS-211LX, Fuji Electronic Industrial Co., Ltd.) at $673 \mathrm{~K}$ for $10 \mathrm{~min}$ under an axial pressure of $55 \mathrm{MPa}$. Highly dense pellets with $\sim 96 \%$ of the theoretical value, were confirmed by directly measured the dimensions and mass of samples and densities as listed in Table S4. The electrical transport properties of Seebeck coefficient $S$ and electrical conductivity $\sigma$ were synchronously measured on the commercial ZEM-3 (Ulvac Riko, Inc.) from $300 \mathrm{~K}$ to $650 \mathrm{~K}$ under the constant helium atmosphere. The densified pellets were coated with a thin layer of electrical insulating and thermal conducting boron nitride about $0.1 \mathrm{~mm}$ to protect the electrode. The total thermal conductivity was calculated from the formula $\kappa=D \times C_{\mathrm{p}} \times d$, where the thermal diffusivity $(D)$ was measured on a Netzsch LFA-457 in the temperature range of 300$650 \mathrm{~K}$, the specific heat $\left(C_{\mathrm{p}}\right)$ was obtained using the approximation of the Dulong-Petit limit, and the density $(d)$ determined by the measured mass and dimensions. The Hall measurement was performed on a home-made apparatus (Tongji University). The carrier concentration $n$ and mobility $\mu$ were calculated by relations of $\mu=\sigma R$ and $n=$ $1 / e R$, where $e$ is the electron charge, $R$ is the Hall efficient, and $\sigma$ is the electrical conductivity. The low-temperature heat capacity and conductivity were measured using a Physical Property Measurement System (PPMS-9: Quantum Design). 
Theoretical Calculation. The density functional theory has been performed to calculate the electronic structures and the density of states for $\mathbf{1}$ and $\mathbf{2}$ with space group of $P 4 / \mathrm{mmm}$ (No. 123) by using the Vienna ab initio Simulation Package (VASP). ${ }^{\mathrm{S} 1-\mathrm{S} 3}$ The generalized gradient approximation $(\mathrm{GGA})^{\mathrm{S} 4}$ was chosen as the exchange correlation functional, and a plane-wave basis with the projector augmented wave $(\mathrm{PAW})^{\mathrm{S} 5}$ potentials were used. The pseudopotentials were used to simulate the ion electron interaction of all constituent elements: Cs $5 s^{2} 5 p^{6} 6 s^{1}, C u 3 d^{10} 4 s^{1}, S 3 s^{2} 3 p^{4}$, Se $4 s^{2} 4 p^{4}$. Structural optimization and the self-consistence calculation of $\mathbf{1}$ and $\mathbf{2}$ was carried out using a dense $0.01 / \AA^{3} k$-point spacing mesh with a kinetic energy cutoff of $500 \mathrm{eV}$. Due to the strongly correlated nature of the materials, the electronic properties of the materials were studied using the DFT + U formalism. ${ }^{\mathrm{S} 6, \mathrm{~S} 7}$ In order to ensure the accuracy of the calculation results, we calculated the $U$ parameter for the DFT $+U$ treatment of $\mathrm{Cu} d$-electrons in $\mathbf{1}$ and $\mathbf{2}$ using the linear response ansatz of Cococcioni et al. ${ }^{\mathrm{S} 8}$ Crystal Orbital Hamilton Populations $(\mathrm{COHP})^{\mathrm{S} 9}$ analysis was carried out by LOBSTER program ${ }^{\mathrm{S} 10, \mathrm{~S} 11}$ to get more in-depth study of chemical bonds. The phonon spectrum was calculated by Phonopy program. ${ }^{\text {S12 }}$

Benefiting from the competitive electrical conductivity $\sigma$, both $\mathrm{CsCu}_{4} \mathrm{~S}_{3}$ and $\mathrm{CsCu}_{4} \mathrm{Se}_{3}$ exhibit the relative large total thermal conductivity $\kappa_{\mathrm{tot}}$ of $3.0-2.5 \mathrm{~W} / \mathrm{m} / \mathrm{K}$ in the range of $300-650 \mathrm{~K}$, as shown in Figure S4. According to the Wiedemann-Franz law $\kappa_{\text {ele }}=$ $L \sigma T$, Lorenz number $L=1.5+\exp \left[\frac{-|S|}{116}\right] \approx 2.36 \times 10^{-8} \mathrm{~W} \Omega \mathrm{K}^{-2},{ }^{, 27}$ we also depict the temperature dependent electrical thermal conductivity with $\kappa_{\text {ele }}$ fall into the range of 2.6-2.3 W/m/K. And the anisotropy in the thermoelectric properties in $\mathrm{CsCu}_{4} \mathrm{Q}_{3}$ is not profoundly large, as given in Figure S11, both the electrical conductivity $\sigma$ in $\mathrm{CsCu}_{4} \mathrm{Q}_{3}$ along perpendicular direction (perp) indicates larger values than the parallel direction (para) pellets. In addition, both the Seebeck coefficient $S$ in $\mathrm{CsCu}_{4} \mathrm{Q}_{3}$ falls into the range of $13-40 \mu \mathrm{V} / \mathrm{K}$ from $300 \mathrm{~K}$ to $650 \mathrm{~K}$ with relative weak anisotropy. Finally, limited by the low $S$ values, $\mathrm{CsCu}_{4} \mathrm{Q}_{3}$ realize the extremely low thermoelectric figure of merit $Z T$ as shown in Figure S11d. 
The average sound velocity $\boldsymbol{v}_{\mathrm{a}}$, Young's modulus $\boldsymbol{E}$, shear modulus $\boldsymbol{G}$, Poisson ratio $\boldsymbol{v}_{\boldsymbol{\rho}}$, Grüneisen parameter $\gamma$, and Debye temperature $\boldsymbol{\theta}_{\boldsymbol{D}}$ are calculated according to the following equations: ${ }^{\mathrm{S} 13-\mathrm{S} 15}$

$$
\begin{gathered}
v_{\mathrm{a}}=\left[\frac{1}{3}\left(\frac{1}{v_{l}^{3}}+\frac{2}{v_{t}^{3}}\right)\right]^{-1 / 3},(\mathrm{~S} 1) \\
E=\frac{\rho v_{t}{ }^{2}\left(3 v_{l}^{2}-4 v_{t}^{2}\right)}{v_{l}{ }^{2}-v_{t}{ }^{2}},(\mathrm{~S} 2) \\
v_{\rho}=\frac{1-2\left(\frac{v_{v}}{v_{l}}\right)}{2-2\left(\frac{v_{t}}{v_{l}}\right)},(\mathrm{S} 3) \\
G=\frac{E}{2\left(1+v_{\rho}\right)},(\mathrm{S} 4) \\
\gamma=\frac{3}{2}\left(\frac{1+v_{\rho}}{2-3 v_{\rho}}\right),(\mathrm{S} 5) \\
\Theta_{D}=\frac{h}{k_{B}}\left[\frac{3 N}{4 \pi V}\right]^{1 / 3} v_{a},(\mathrm{~S} 6)
\end{gathered}
$$

The glass limit of lattice thermal conductivity can be obtained via the measured longitudinal and transverse sound velocities ( $\boldsymbol{v}_{\mathbf{l}}$ and $\boldsymbol{v}_{\mathrm{t}}$ ), briefly guided by Cahill's formula:S16

$$
\kappa_{\text {limit }}=\frac{1}{2}\left[\left(\frac{\pi}{6}\right)^{1 / 3}\right] k_{B} V^{-2 / 3}\left(2 v_{t}+v_{l}\right),
$$

The Debye-Callaway model:S17,S18

$$
\kappa_{\text {lat }}=\frac{k_{\mathrm{B}}}{2 \pi^{2} v_{a}}\left(\frac{k_{\mathrm{B}}}{\hbar}\right)^{3} T^{3} \int_{0}^{\Theta_{D} / T} \frac{x^{4} e^{x}}{\tau_{c}^{-1}\left(e^{x}-1\right)^{2}} d x
$$

where $x=\hbar \omega / k_{\mathrm{B}} T, \hbar, \omega$, and $k_{\mathrm{B}}$ are the reduced Planck constant, phonon angular frequency, and Boltzmann constant. $v_{\mathrm{a}}$ is the average sound velocity, $\tau_{c}$ is the phonon relaxation time, containing a series of scattering mechanisms, such as grain boundary scattering, point defect scattering, and phonon-phonon Umklapp scattering, their relaxation time can be written as follows:

$$
\begin{gathered}
\tau_{B}^{-1}=\frac{v_{a}}{L},(\mathrm{~S} 9) \\
\tau_{D}^{-1}=\frac{V}{4 \pi v_{a}{ }^{3}} \omega^{4} \sum f_{i}\left(\frac{\bar{m}-m_{i}}{\bar{m}}\right)^{2},(\mathrm{~S} 10) \\
\tau_{U}^{-1}=\frac{h \gamma_{G}{ }^{2} \omega^{2} T}{M v^{2} \square_{D}} \mathrm{e}^{-\square_{D} / 3 T}(\mathrm{~S} 11)
\end{gathered}
$$


Where $L$ is the average grain size, $V$ is the unit cell volume, $\gamma_{G}$ is the Grüneisen parameter, $m_{\mathrm{i}}$ is the atomic mass, $\bar{m}$ is the atomic average mass, and $f_{i}$ is the proportion factor, $M$ is the average mass of unit cell.
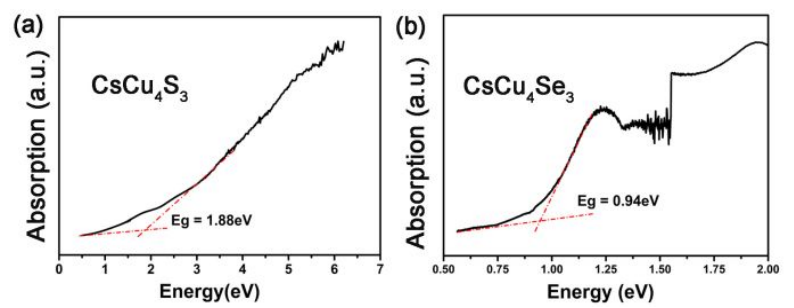

Figure S1. The UV-Vis spectra for $\mathrm{CsCu}_{4} \mathrm{~S}_{3}$ and $\mathrm{CsCu}_{4} \mathrm{Se}_{3}$.
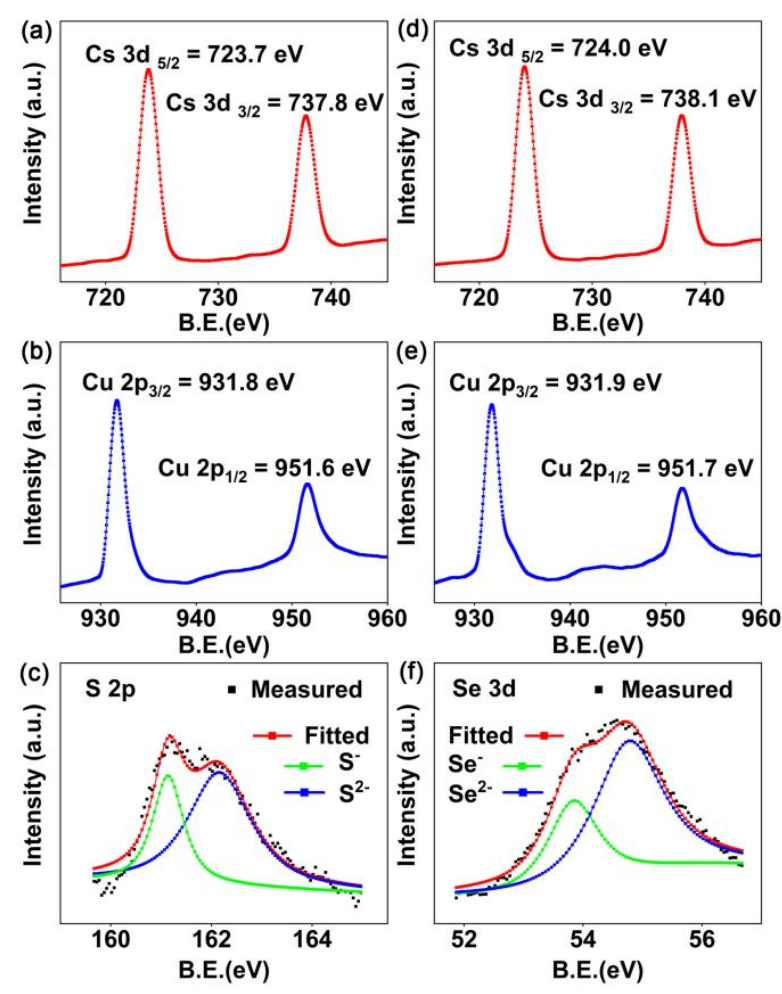

Figure S2. X-ray photoelectron spectroscopy of 1 (a)-(c) and 2 (d)-(f). Spectra curve for $\mathrm{Cs} 3 \mathrm{~d}, \mathrm{Cu} 2 \mathrm{p}, \mathrm{S} 2 \mathrm{p}$, and the Se $3 \mathrm{~d}$ core levels, respectively. 

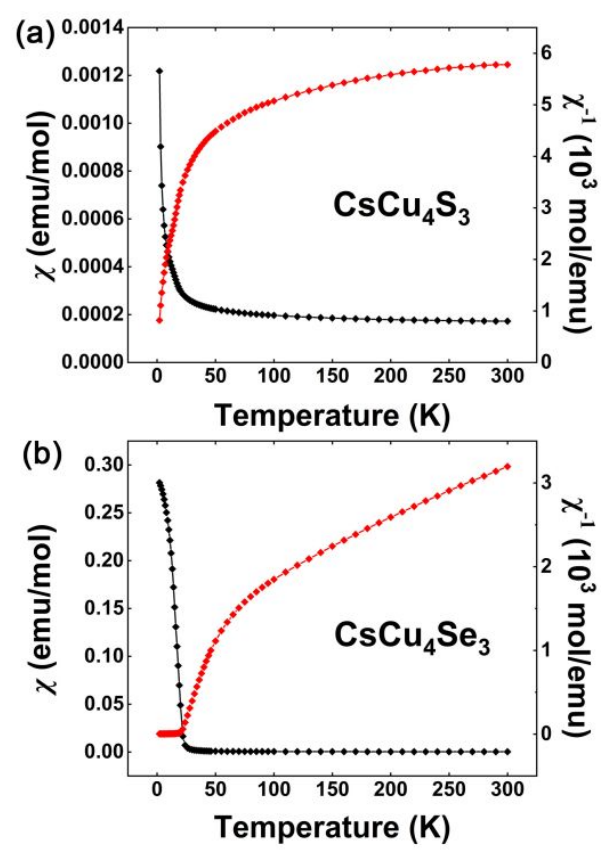

Figure S3. Temperature dependence of magnetic susceptibility and inverse susceptibility of $\mathrm{CsCu}_{4} \mathrm{~S}_{3}$ (a) and $\mathrm{CsCu}_{4} \mathrm{Se}_{3}$ (b) measured in an external field of 1000 Oe.
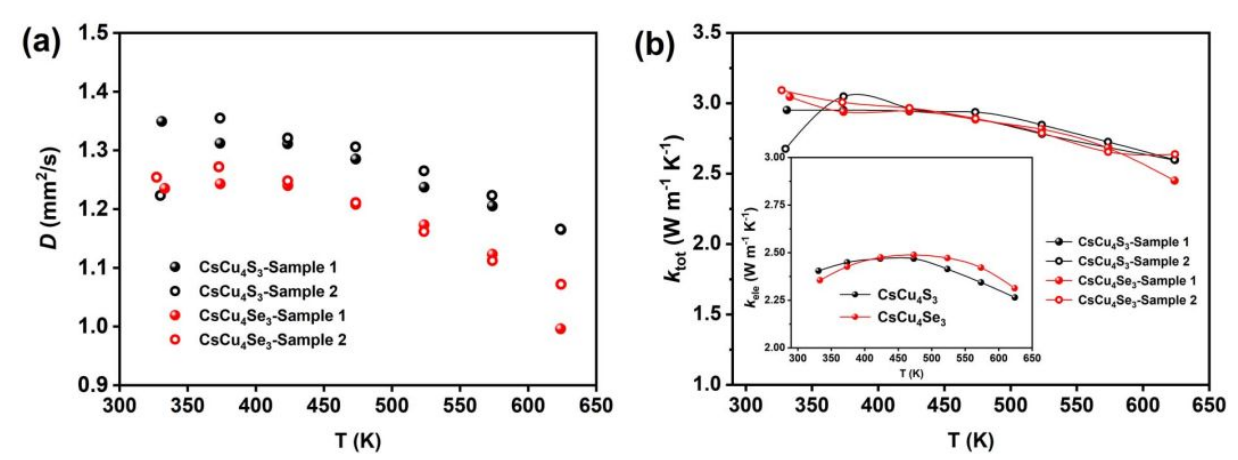

Figure S4. (a) The temperature-dependent thermal diffusivities, (b) total thermal conductivity $\left(\kappa_{\text {tot }}\right)$ and electrical thermal conductivity $\left(\kappa_{\text {ele }}\right)$ of $\mathrm{CsCu}_{4} \mathrm{Q}_{3}(\mathrm{Q}=\mathrm{S}, \mathrm{Se})$ along the perpendicular direction. The Lorenz number $(L)$ was estimated to be $10^{-8} \mathrm{~W}$ $\Omega \mathrm{K}^{-2}$ according to the equation ${ }^{[\mathrm{S} 27]}: L \approx 1.5+\exp \left(-\frac{|S|}{116 \mu \mathrm{V} / \mathrm{K}}\right) \approx 2.36 \times 10^{-8} \mathrm{~W}$ $\Omega \mathrm{K}^{-2}$. 


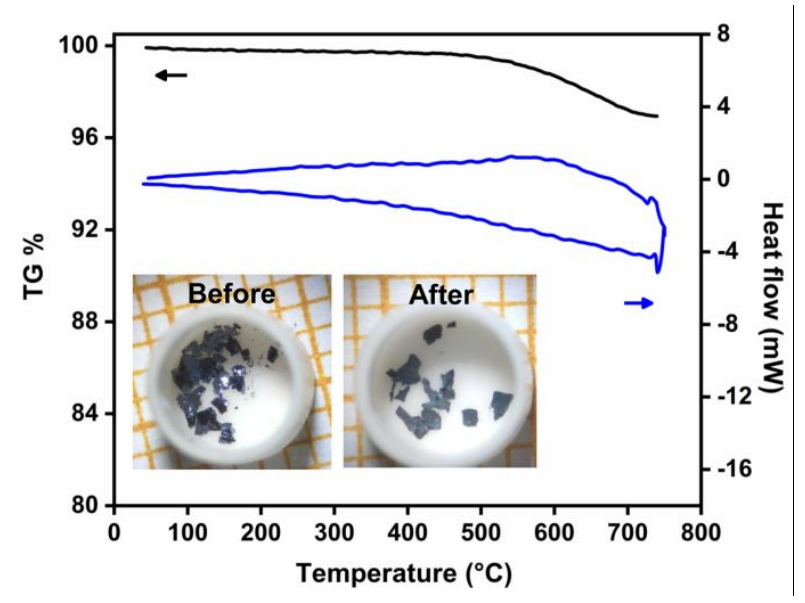

Figure S5. TG (black) and DSC (blue) diagram, and photographs of as-synthesized of $\mathrm{CsCu}_{4} \mathrm{Se}_{3}$ before and after the TG-DSC measurement.

Thanks to the response-function language, we have the effective interaction parameter $U(6.72 \mathrm{eV}$ for $\mathbf{1}, 7.26 \mathrm{eV}$ for 2$)$ associated to site $I$ based on the equation: ${ }^{\mathrm{S} 8}$

$$
U=x^{-1}-x_{0}^{-1} \approx\left(\frac{\partial N_{I}^{\mathrm{SCF}}}{\partial V_{I}}\right)^{-1}-\left(\frac{\partial N_{I}^{\mathrm{NSCF}}}{\partial V_{I}}\right)^{-1}
$$
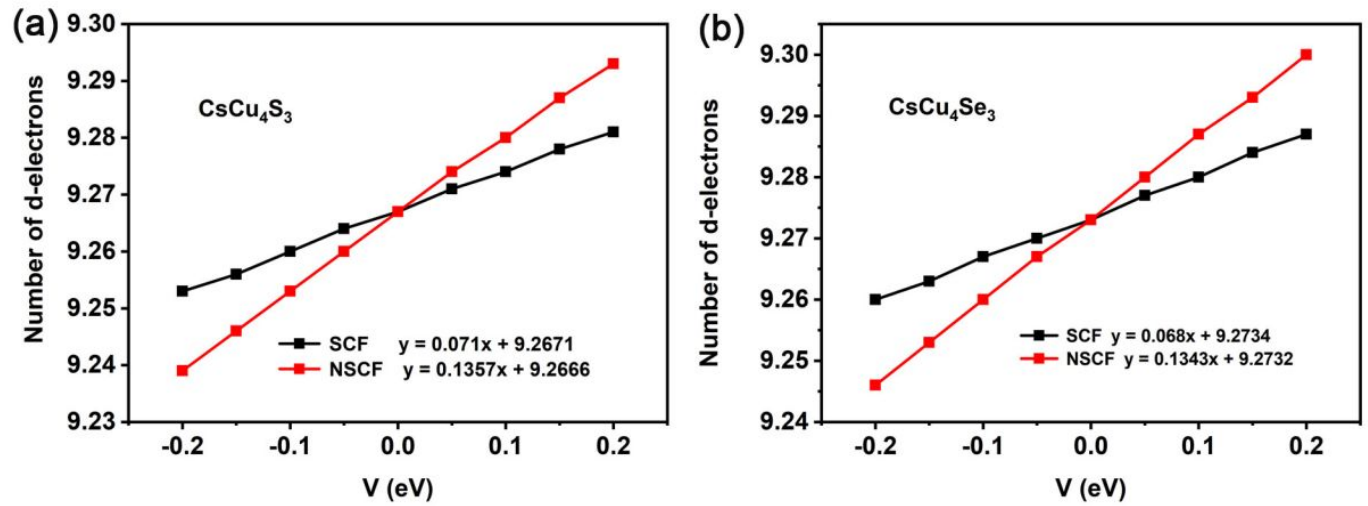

Figure S6. The linear fit of the number of $d$-electrons on $\mathrm{Cu}$ ( $4 i$ site) as a function of the additional potential $\mathrm{V}(\mathrm{eV})$ in $\mathrm{CsCu}_{4} \mathrm{~S}_{3}$ (a) and $\mathrm{CsCu}_{4} \mathrm{Se}_{3}$ (b). 


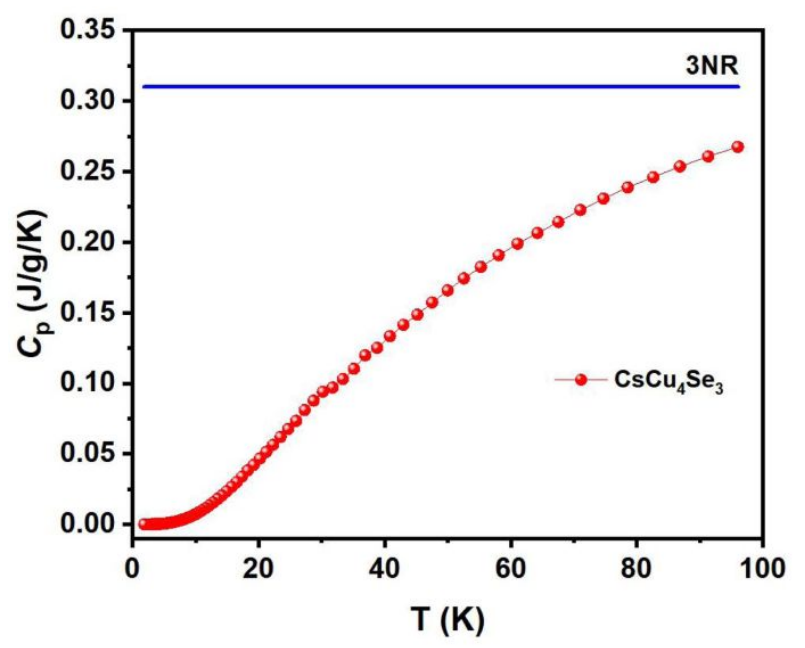

Figure S7. Temperature dependence of heat capacity $C_{\mathrm{p}}$ (red dots) in the range of 0 $100 \mathrm{~K}$. The Dulong-Petit value (blue line) for the high temperature heat capacity in the solid $\mathrm{CsCu}_{4} \mathrm{Se}_{3}$.
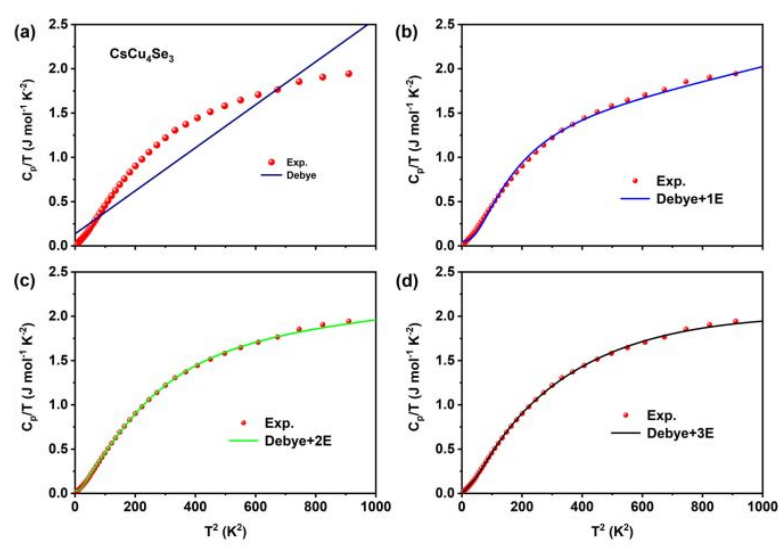

Figure S8. The experimental $C_{\mathrm{p}} / T$ with respect to $\mathrm{T}^{2}$ at low temperature fitted by a combination of Debye and multiple Einstein models. Red dots illustrate the experimental heat capacity plotted as $C_{\mathrm{p}} / T$ versus $\mathrm{T}^{2}$ for $\mathrm{CsCu}_{4} \mathrm{Se}_{3}$. 

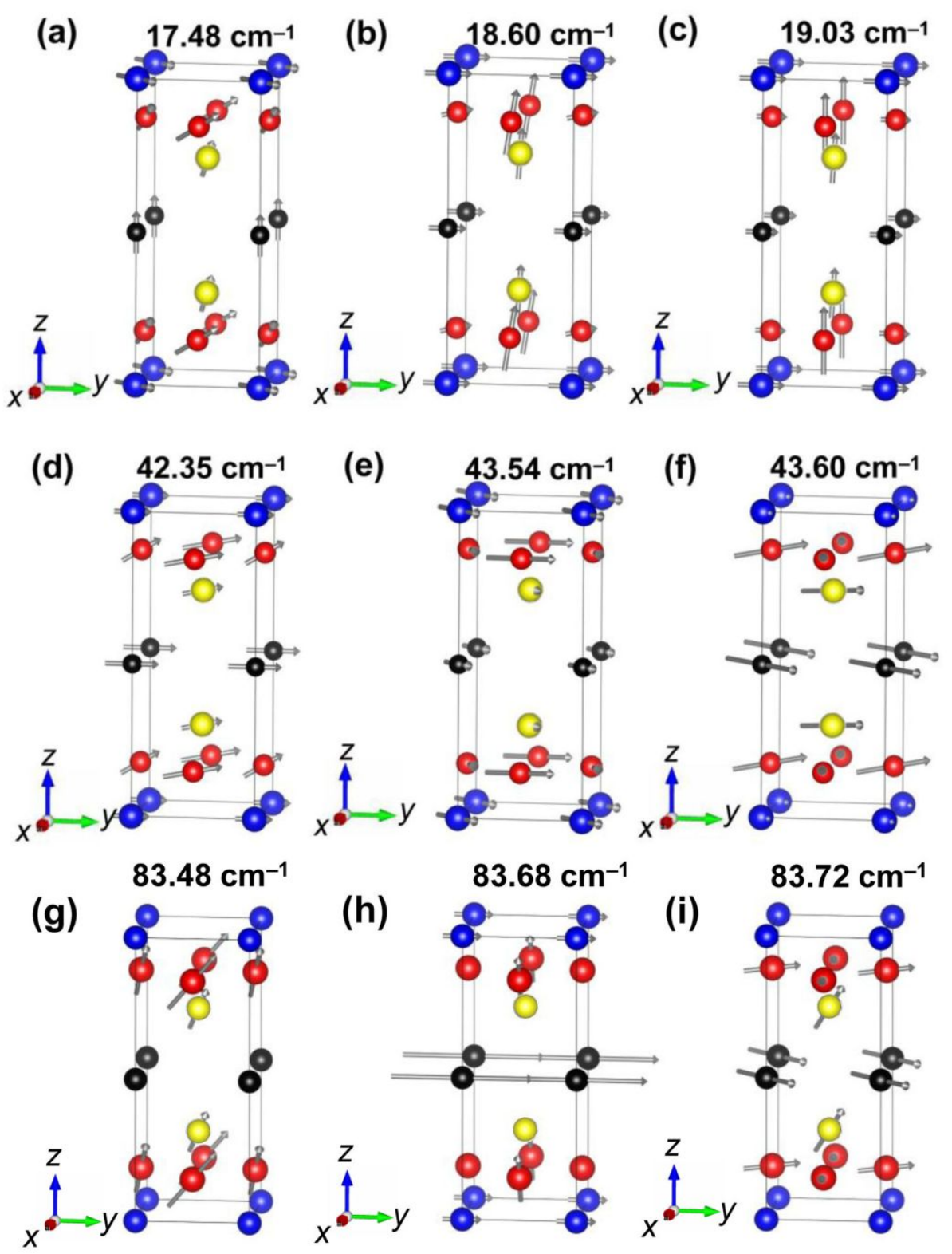

(h)

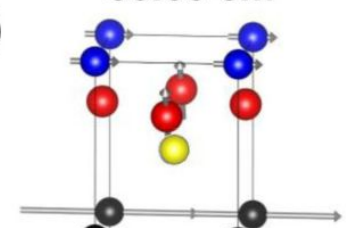

(i)
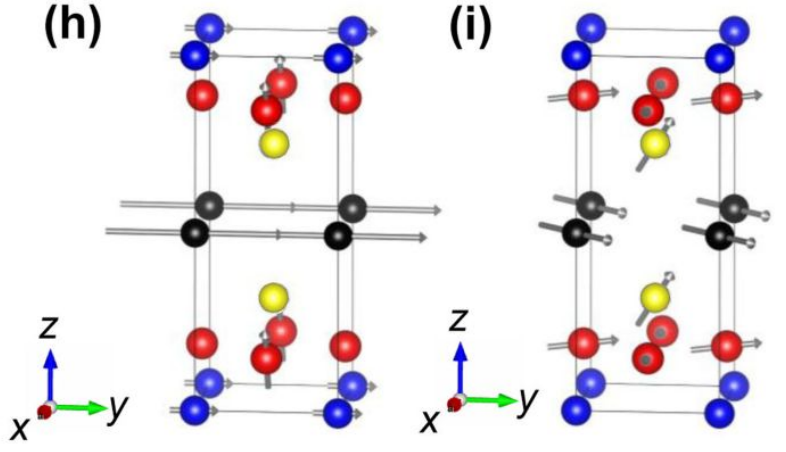

Figure S9. Eigen vector visualizations for the phonon modes around $(\mathrm{a}-\mathrm{c}) 18.94 \mathrm{~cm}^{-1}$, (d-f) $43.47 \mathrm{~cm}^{-1}$, and (g-i) $83.61 \mathrm{~cm}^{-1}$. Black: $\mathrm{Cs}^{+}$, red: $\mathrm{Cu}^{+}$, blue: $\mathrm{Se}^{-}$, yellow: $\mathrm{Se}^{2-}$. 

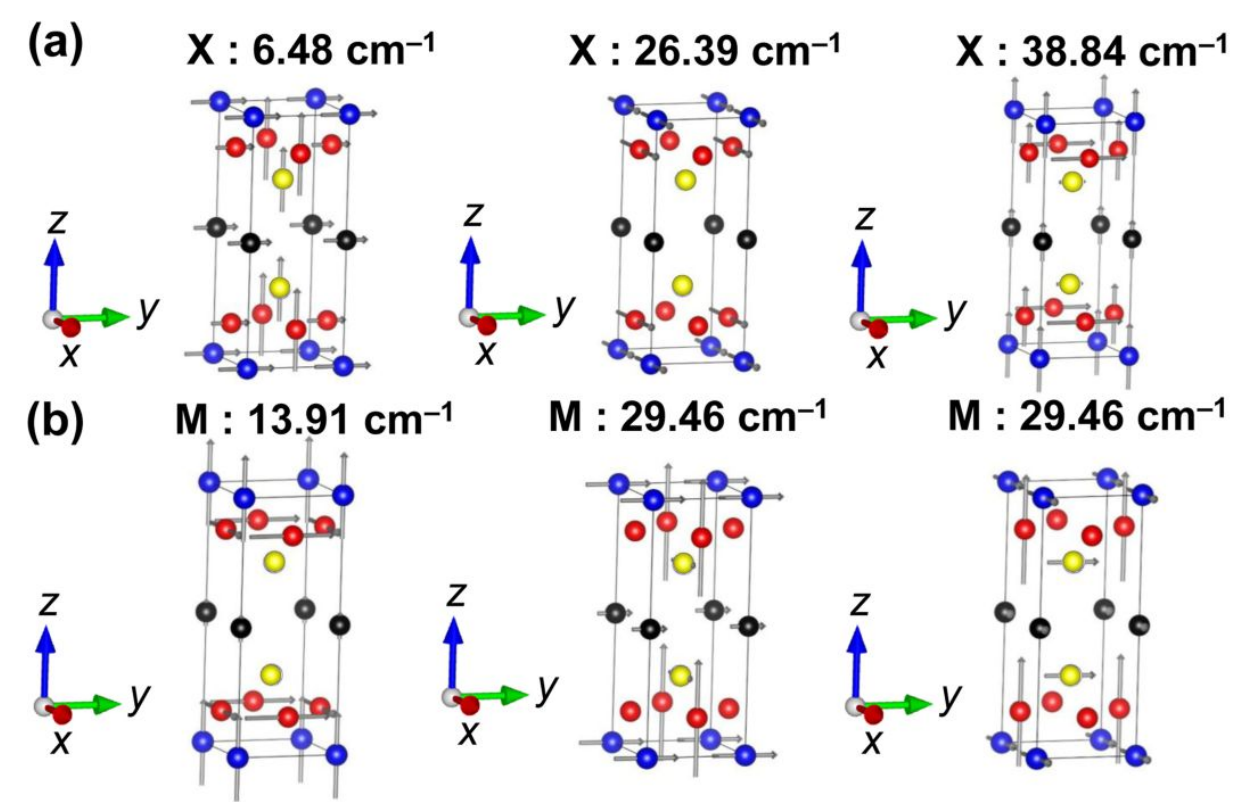

$M: 29.46 \mathrm{~cm}^{-1}$

$M: 29.46 \mathrm{~cm}^{-1}$
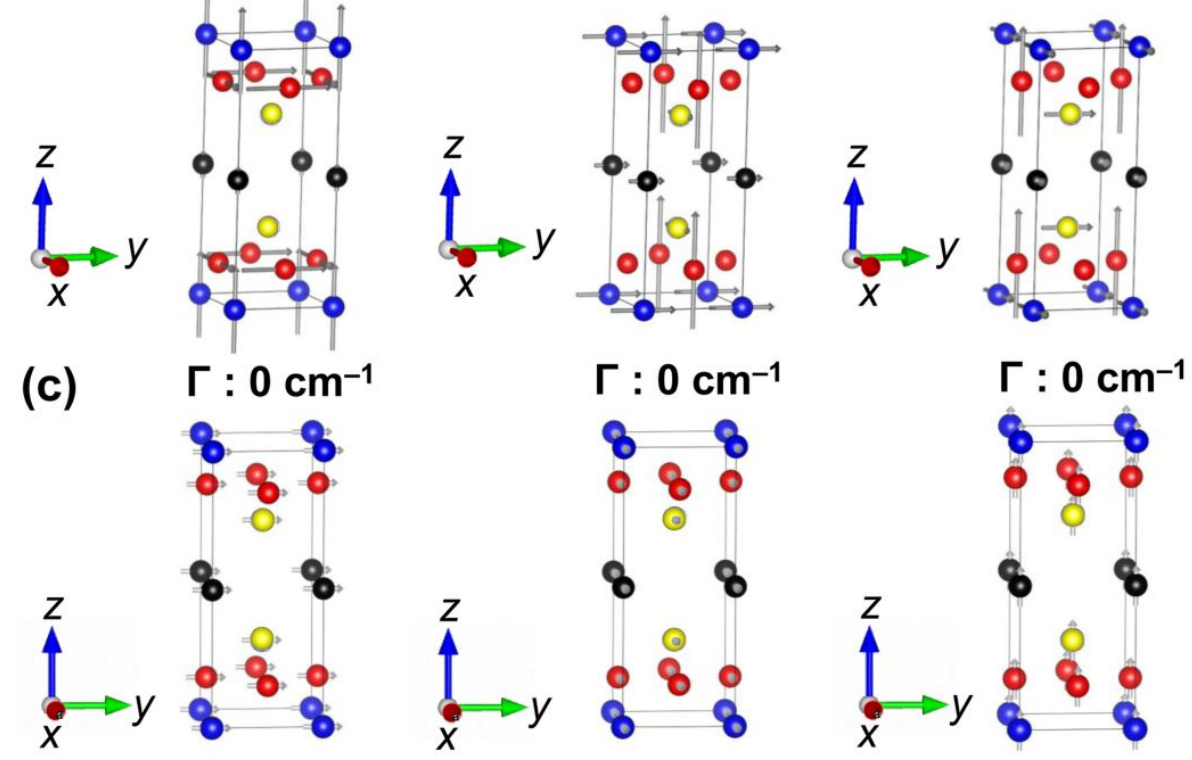

Figure S10. Eigen vector visualizations for the phonon modes at (a) X $\left(6.48 \mathrm{~cm}^{-1}\right.$, $\left.26.39 \mathrm{~cm}^{-1}, 38.84 \mathrm{~cm}^{-1}\right)$, (b) $\mathrm{M}\left(13.91 \mathrm{~cm}^{-1}, 29.46 \mathrm{~cm}^{-1}, 29.46 \mathrm{~cm}^{-1}\right)$, and $\Gamma\left(0 \mathrm{~cm}^{-1}, 0\right.$ $\mathrm{cm}^{-1}, 0 \mathrm{~cm}^{-1}$ ) points, respectively. Black: $\mathrm{Cs}^{+}$, red: $\mathrm{Cu}^{+}$, blue: $\mathrm{Se}^{-}$, yellow: $\mathrm{Se}^{2-}$. 

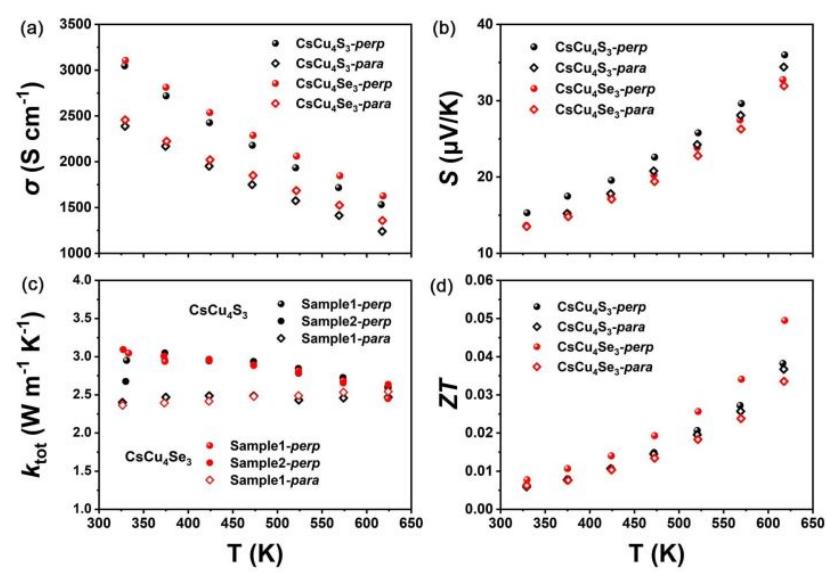

Figure S11. Temperature-dependent (a) electrical conductivity, (b) Seebeck coefficient, (c) total thermal conductivity, and (d) the figure of merit $Z T$ in $\mathrm{CsCu}_{4} \mathrm{Q}_{3}(\mathrm{Q}=\mathrm{S}, \mathrm{Se})$ along perpendicular (perp) and parallel (para) directions.
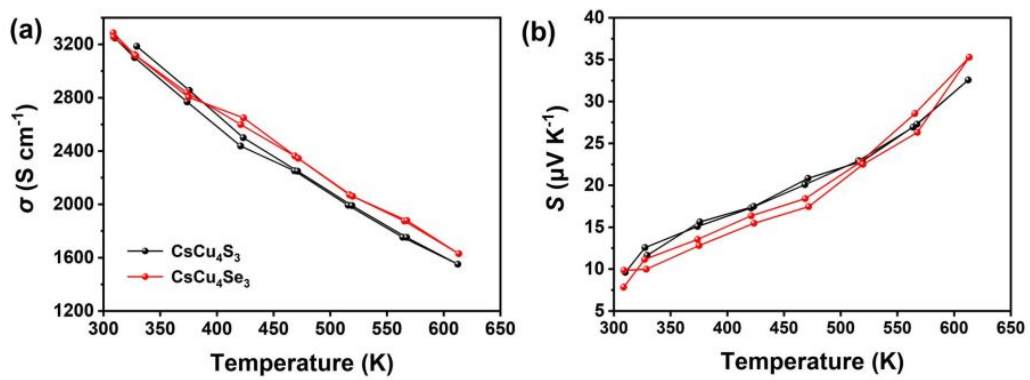

Figure S12. The recyclability of the electrical conductivity $\sigma$ and Seebeck coefficient $S$ of (a) $\mathrm{CsCu}_{4} \mathrm{~S}_{3}$ and (b) $\mathrm{CsCu}_{4} \mathrm{Se}_{3}$ that simultaneously measured on the ZEM-3, indicating their good stability with minor deviation in the cooling data.

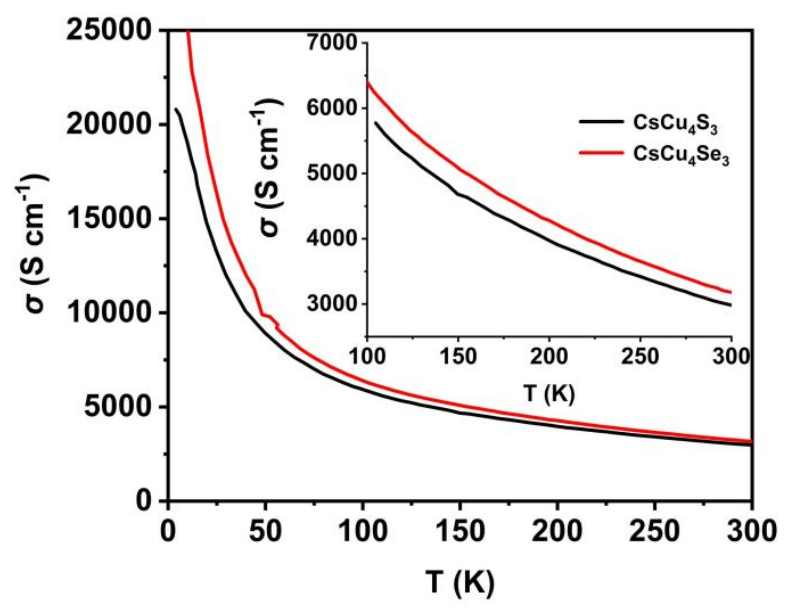

Figure S13. The low temperature electrical conductivity of $\mathrm{CsCu}_{4} \mathrm{~S}_{3}$ (black line) and $\mathrm{CsCu}_{4} \mathrm{Se}_{3}$ (red line). 
Table S1. Crystallographic Data and Refinement Details for $\mathrm{CsCu}_{4} \mathrm{~S}_{3}$ and $\mathrm{CsCu}_{4} \mathrm{Se}_{3}$ at Room Temperature.

\begin{tabular}{|c|c|c|}
\hline Formula & $\mathrm{CsCu}_{4} \mathrm{~S}_{3}$ & $\mathrm{CsCu}_{4} \mathrm{Se}_{3}$ \\
\hline formula weight & 483.32 & 623.95 \\
\hline crystal system & Tetragonal & Tetragonal \\
\hline crystal color & Black & Black \\
\hline space group & P4/mmm (No. 123) & P4/mmm (No. 123) \\
\hline$a(\AA)$ & $3.9759(3)$ & $4.093(2)$ \\
\hline$b(\AA)$ & $3.9759(3)$ & $4.093(2)$ \\
\hline$c(\AA)$ & $9.6950(11)$ & $10.076(5)$ \\
\hline$\alpha\left(^{\circ}\right)$ & 90 & 90 \\
\hline$V\left(\AA^{3}\right)$ & $153.26(3)$ & $168.80(19)$ \\
\hline$Z$ & 1 & 1 \\
\hline$D_{c}\left(\mathrm{~g} / \mathrm{cm}^{3}\right)$ & 5.237 & 6.138 \\
\hline$\mu\left(\mathrm{mm}^{-1}\right)$ & 20.421 & 33.786 \\
\hline GOOF on $F^{2}$ & 1.138 & 1.177 \\
\hline$R_{1}, w R_{2}(I>2 \sigma(I))^{\mathrm{a}}$ & $0.0394,0.0793$ & $0.0487,0.0971$ \\
\hline$R_{1}, w R_{2}$ (all data) & $0.0417,0.0810$ & $0.0618,0.1023$ \\
\hline largest diff. peak and hole $\left(\mathrm{e} / \AA^{3}\right)$ & $1.785,-1.502$ & $1.507,-2.372$ \\
\hline
\end{tabular}

${ }^{\mathrm{a}} R_{1}=\Sigma|| F_{\mathrm{o}}|-| F_{\mathrm{c}}|| \Sigma\left|\mathrm{F}_{\mathrm{o}}\right|, \mathrm{w} R_{2}=\left[\Sigma w\left(F_{\mathrm{o}}{ }^{2}-F_{\mathrm{c}}{ }^{2}\right)^{2} / \Sigma w\left(F_{\mathrm{o}}{ }^{2}\right)^{2}\right]^{1 / 2}$ 
Table S2. Atomic Coordinates and Equivalent Isotropic Displacement Parameters of $\mathrm{CsCu}_{4} \mathrm{~S}_{3}$ and $\mathrm{CsCu}_{4} \mathrm{Se}_{3}$.

\begin{tabular}{cccccccc}
\hline \multicolumn{7}{c}{$\mathbf{C s C u}_{4} \mathbf{S}_{3}$} \\
\hline Atom & Wyckoff site & Oxidation & $\mathbf{x}$ & $\boldsymbol{y}$ & $\boldsymbol{z}$ & $\boldsymbol{U}(\mathbf{e q})\left(\AA^{2}\right)^{*}$ & Occu. \\
$\mathrm{Cs} 1$ & $1 a$ & +1 & 0.0000 & 0.0000 & 0.0000 & $0.017(1)$ & 1 \\
$\mathrm{Cu} 1$ & $4 i$ & +1 & 0.0000 & 0.5000 & $-0.3494(1)$ & $0.023(1)$ & 1 \\
$\mathrm{~S} 1$ & $1 b$ & -1 & 0.0000 & 0.0000 & -0.5000 & $0.013(1)$ & 1 \\
$\mathrm{~S} 2$ & $2 h$ & -2 & -0.5000 & 0.5000 & $-0.2271(3)$ & $0.013(1)$ & 1 \\
\hline \multicolumn{7}{c}{$\mathbf{C s C u}_{4} \mathbf{S e}_{\mathbf{3}}$} \\
\hline Atom & Wyckoff site & $\mathbf{O x i d a t i o n}$ & $\mathbf{x}$ & $\boldsymbol{y}$ & $\boldsymbol{z}$ & $\boldsymbol{U}(\mathbf{e q})\left(\AA^{2}\right)^{*}$ & Occu. \\
$\mathrm{Cs}$ & $1 b$ & +1 & 0.0000 & 0.0000 & -0.5000 & $0.019(1)$ & 1 \\
$\mathrm{Cu} 1$ & $4 i$ & +1 & 0.0000 & 0.5000 & $-0.1514(2)$ & $0.025(1)$ & 1 \\
$\mathrm{Se} 1$ & $1 a$ & -1 & 0.0000 & 0.0000 & 0.0000 & $0.014(1)$ & 1 \\
$\mathrm{Se} 2$ & $2 h$ & -2 & -0.5000 & 0.5000 & $-0.2798(2)$ & $0.015(1)$ & 1 \\
\hline
\end{tabular}

Table S3. Bond Lengths $(\AA)$ and Bond Angles (deg) for $\mathrm{CsCu}_{4} \mathrm{~S}_{3}$ and $\mathrm{CsCu}_{4} \mathrm{Se}_{3}$.

\begin{tabular}{|c|c|c|c|}
\hline \multicolumn{2}{|c|}{$\mathrm{CsCu}_{4} \mathrm{~S}_{3}$} & \multicolumn{2}{|c|}{$\mathrm{CsCu}_{4} \mathrm{Se}_{3}$} \\
\hline $\mathrm{Cu} 1-\mathrm{S} 1\left(\mathrm{~S}^{-}\right)$ & 2.4667 & $\mathrm{Cu} 1-\mathrm{Se} 1\left(\mathrm{Se}^{-}\right)$ & 2.5527 \\
\hline $\mathrm{Cu} 1-\mathrm{S} 1\left(\mathrm{~S}^{-}\right)$ & 2.4667 & $\mathrm{Cu} 1-\mathrm{Se} 1\left(\mathrm{Se}^{-}\right)$ & 2.5527 \\
\hline $\mathrm{Cu} 1-\mathrm{S} 2\left(\mathrm{~S}^{2-}\right)$ & 2.3146 & $\mathrm{Cu} 1-\mathrm{Se} 2\left(\mathrm{Se}^{2-}\right)$ & 2.4212 \\
\hline $\mathrm{Cu} 1-\mathrm{S} 2\left(\mathrm{~S}^{2-}\right)$ & 2.3146 & $\mathrm{Cu} 1-\mathrm{Se} 2\left(\mathrm{Se}^{2-}\right)$ & 2.4212 \\
\hline $\mathrm{Cs} 1-\mathrm{S} 2\left(\mathrm{~S}^{2-}\right)$ & 3.5709 & $\mathrm{Cs} 1-\mathrm{Se} 2\left(\mathrm{Se}^{2-}\right)$ & 3.6472 \\
\hline $\mathrm{S} 1\left(\mathrm{~S}^{-}\right)-\mathrm{Cu} 1-\mathrm{S} 1\left(\mathrm{~S}^{-}\right)$ & 107.40 & $\mathrm{Se} 1\left(\mathrm{Se}^{-}\right)-\mathrm{Cu} 1-\mathrm{Se} 1\left(\mathrm{Se}^{-}\right)$ & 106.58 \\
\hline $\mathrm{S} 1\left(\mathrm{~S}^{-}\right)-\mathrm{Cu} 1-\mathrm{S} 2\left(\mathrm{~S}^{2-}\right)$ & 107.65 & $\mathrm{Se} 1\left(\mathrm{Se}^{-}\right)-\mathrm{Cu} 1-\mathrm{Se} 2\left(\mathrm{Se}^{2-}\right)$ & 108.62 \\
\hline $\mathrm{S} 2\left(\mathrm{~S}^{2-}\right)-\mathrm{Cu} 1-\mathrm{S} 2\left(\mathrm{~S}^{2-}\right)$ & 118.38 & $\mathrm{Se} 2\left(\mathrm{Se}^{2-}\right)-\mathrm{Cu} 1-\mathrm{Se} 2\left(\mathrm{Se}^{2-}\right)$ & 115.41 \\
\hline
\end{tabular}

Table S4. Densities of titled compounds $\mathrm{CsCu}_{4} \mathrm{~S}_{3}$ and $\mathrm{CsCu}_{4} \mathrm{Se}_{3}$.

\begin{tabular}{cccc}
\hline Sample & Expt. Density $\left(\mathbf{g} / \mathbf{c m}^{3}\right)$ & Calc. Density $\left(\mathbf{g} / \mathbf{c m}^{3}\right)$ & \\
\hline $\mathrm{CsCu}_{4} \mathrm{~S}_{3}$ & 5.16 & 5.237 & $98 \%$ \\
$\mathrm{CsCu}_{4} \mathrm{Se}_{3}$ & 5.87 & 6.138 & $96 \%$ \\
\hline
\end{tabular}


Table S5. Valence states in $\mathrm{CsCu}_{4} \mathrm{~S}_{3}, \mathrm{CsCu}_{4} \mathrm{Se}_{3}, \alpha-\mathrm{CsCu}_{5} \mathrm{Se}_{3},{ }^{\mathrm{S} 19} \mathrm{Na}_{3} \mathrm{Cu}_{4} \mathrm{~S}_{4},{ }^{\mathrm{S} 20}$ $\mathrm{CsAg}_{5} \mathrm{Te}_{3},{ }^{\mathrm{S} 21} \mathrm{NaCu}_{4} \mathrm{Se}_{3},{ }^{\mathrm{S} 22} \mathrm{NaCu}_{6} \mathrm{Se}_{4},{ }^{\mathrm{S} 23}$ and $\mathrm{NaCu}_{4} \mathrm{Se}_{4} \cdot{ }^{\mathrm{S} 24}$

\begin{tabular}{cc}
\hline Compound & \\
\hline $\mathrm{CsCu}_{4} \mathrm{~S}_{3}$ & $\left(\mathrm{Cs}^{+}\right)\left(\mathrm{Cu}^{+}\right)_{4}\left(\mathrm{~S}^{2-}\right)_{2}\left(\mathrm{~S}^{-}\right)$ \\
$\mathrm{CsCu}_{4} \mathrm{Se}_{3}$ & $\left(\mathrm{Cs}^{+}\right)\left(\mathrm{Cu}^{+}\right)_{4}\left(\mathrm{Se}^{2-}\right)_{2}\left(\mathrm{Se}^{-}\right)$ \\
$\alpha-\mathrm{CsCu}_{5} \mathrm{Se}_{3}$ & $\left(\mathrm{Cs}^{+}\right)\left(\mathrm{Cu}^{+}\right)_{5}\left(\mathrm{Se}^{2-}\right)_{3}$ \\
$\mathrm{Na}_{3} \mathrm{Cu}_{4} \mathrm{~S}_{4}$ & $\left(\mathrm{Na}^{+}\right)_{3}\left(\mathrm{Cu}^{+}\right)_{4}\left(\mathrm{~S}^{2-}\right)_{3}\left(\mathrm{~S}^{-}\right)$ \\
$\mathrm{CsAg}_{5} \mathrm{Te}_{3}$ & $\left(\mathrm{Cs}^{+}\right)\left(\mathrm{Ag}^{+}\right)_{5}\left(\mathrm{Te}^{2-}\right)_{3}$ \\
$\mathrm{NaCu}_{4} \mathrm{Se}_{3}$ & $\left(\mathrm{Na}^{+}\right)\left(\mathrm{Cu}^{+}\right)_{4}\left(\mathrm{Se}^{2-}\right)_{2}\left(\mathrm{Se}^{-}\right)$ \\
$\mathrm{NaCu}_{6} \mathrm{Se}_{4}$ & $\left(\mathrm{Na}^{+}\right)\left(\mathrm{Cu}^{+}\right)_{6}\left(\mathrm{Se}^{2-}\right)_{3}\left(\mathrm{Se}^{-}\right)$ \\
$\mathrm{NaCu}_{4} \mathrm{Se}_{4}$ & $\left(\mathrm{Na}^{+}\right)\left(\mathrm{Cu}^{+}\right)_{4}\left(\mathrm{Se}^{2-}\right)\left(\mathrm{Se}^{-}\right)\left(\mathrm{Se}_{2}\right)^{2-}$ \\
\hline
\end{tabular}

Table S6. Room temperature electrical conductivity $\sigma$ and Seebeck coefficient $S$ of $\mathrm{CsCu}_{4} \mathrm{~S}_{3}, \quad \mathrm{CsCu}_{4} \mathrm{Se}_{3}, \quad \alpha-\mathrm{CsCu}_{5} \mathrm{Se}_{3}, \mathrm{~s}^{2} 19 \mathrm{CsAg}_{5} \mathrm{Te}_{3}, \mathrm{~s} 21 \quad \mathrm{NaCu}_{4} \mathrm{Se}_{3}, \mathrm{~s} 22 \quad \mathrm{NaCu}_{6} \mathrm{Se}_{4}, \mathrm{~s} 23$ $\mathrm{NaCu}_{4} \mathrm{Se}_{4},{ }^{\mathrm{S} 24} o-\mathrm{CsCu}_{5} \mathrm{~S}_{3},{ }^{\mathrm{S} 25}$ and $\mathrm{Cs}_{3} \mathrm{Cu}_{20} \mathrm{Te}_{13} .{ }^{\mathrm{S} 26}$

\begin{tabular}{cccc}
\hline Compound & $\sigma(\mathbf{S} / \mathbf{c m})$ & $\boldsymbol{S}(\boldsymbol{\mu} \mathbf{V} / \mathbf{K})$ & Ref \\
$\mathrm{CsCu}_{4} \mathrm{~S}_{3}$ & 3046 & 15 & this work \\
$\mathrm{CsCu}_{4} \mathrm{Se}_{3}$ & 3106 & 13 & this work \\
$\alpha-\mathrm{CsCu}_{5} \mathrm{Se}_{3}$ & 420 & 25 & {$[\mathrm{~S} 19]$} \\
$\mathrm{CsAg}_{5} \mathrm{Te}_{3}$ & 0.17 & 600 & {$[\mathrm{~S} 21]$} \\
$\mathrm{NaCu}_{4} \mathrm{Se}_{3}$ & 300 & 10 & {$[\mathrm{~S} 22]$} \\
$\mathrm{NaCu}_{6} \mathrm{Se}_{4}$ & 3000 & 5 & {$[\mathrm{~S} 23]$} \\
$\mathrm{NaCu}_{4} \mathrm{Se}_{4}$ & 8547 & - & {$[\mathrm{S} 24]$} \\
$o-\mathrm{CsCu}_{5} \mathrm{~S}_{3}$ & 16 & 120 & {$[\mathrm{~S} 25]$} \\
$\mathrm{Cs}_{3} \mathrm{Cu}_{20} \mathrm{Te}_{13}$ & 2390 & - & {$[\mathrm{S} 26]$} \\
\hline
\end{tabular}

Table S7. Comparison of the carrier concentration $n$ and the carrier mobility $\mu$ at room temperature for related chalcogenides, $\mathrm{CsCu}_{4} \mathrm{~S}_{3}, \mathrm{CsCu}_{4} \mathrm{Se}_{3}, \quad \alpha-\mathrm{CsCu}_{5} \mathrm{Se}_{3},{ }^{\mathrm{S} 19}$ $\mathrm{CsAg}_{5} \mathrm{Te}_{3},{ }^{\mathrm{S} 21} \mathrm{NaCu}_{4} \mathrm{Se}_{3},{ }^{\mathrm{S} 22} \mathrm{NaCu}_{6} \mathrm{Se}_{4},{ }^{\mathrm{S} 23}$ and $\mathrm{NaCu}_{4} \mathrm{Se}_{4}$. 224

\begin{tabular}{cccc}
\hline Compound & $\boldsymbol{n}_{\mathbf{H}}\left(\mathbf{c m}^{-3}\right)$ & $\boldsymbol{\mu}_{\mathbf{H}}\left(\mathbf{c m}^{\mathbf{2}} \mathbf{V}^{-\mathbf{1}} \mathbf{S}^{-\mathbf{1}}\right)$ & Ref \\
\hline $\mathrm{CsCu}_{4} \mathrm{~S}_{3}$ & $2.75 \times 10^{21}$ & 6.6 & this work \\
$\mathrm{CsCu}_{4} \mathrm{Se}_{3}$ & $3.71 \times 10^{21}$ & 5.0 & this work \\
$\alpha-\mathrm{CsCu}_{5} \mathrm{Se}_{3}$ & $2.73 \times 10^{20}$ & 5.3 & {$[\mathrm{~S} 19]$} \\
$\mathrm{CsAg}_{5} \mathrm{Te}_{3}$ & $1.01 \times 10^{17}$ & 14 & {$[\mathrm{~S} 21]$} \\
$\mathrm{NaCu}_{4} \mathrm{Se}_{3}$ & $6.12 \times 10^{21}$ & 0.3 & {$[\mathrm{~S} 22]$} \\
$\mathrm{NaCu}_{6} \mathrm{Se}_{4}$ & $2.83 \times 10^{21}$ & 8.7 & {$[\mathrm{~S} 23]$} \\
$\mathrm{NaCu}_{4} \mathrm{Se}_{4}$ & $5.39 \times 10^{21}$ & 11 & {$[\mathrm{~S} 24]$} \\
\hline
\end{tabular}


Table S8. Parameters obtained from fitting the experimental $C_{\mathrm{p}} / T-T^{2}$ plot for $\mathrm{CsCu}_{4} \mathrm{Se}_{3}$ when introducing different number of Einstein oscillators in the combined Debye-Einstein model.

\begin{tabular}{ccccc}
\hline Parameter & Debye & Debye+1E & Debye+2E & Debye+3E \\
\hline$\gamma / \mathrm{J} \mathrm{mol}^{-1} \mathrm{~K}^{-2}$ & 0.1356 & 0.0434 & 0.0262 & 0.0221 \\
$\beta / \mathrm{J} \mathrm{mol}^{-1} \mathrm{~K}^{-4}$ & 0.0024 & 0.001 & 0.0005 & 0.0002 \\
$\mathbf{A}_{\mathbf{1}} / \mathrm{J} \mathrm{mol}^{-1} \mathrm{~K}^{-1}$ & $/$ & 42.08 & 65.38 & 2.5 \\
$\boldsymbol{\Theta}_{\boldsymbol{E} \mathbf{1}} / \mathbf{K}$ & $/$ & 63.39 & 84.44 & $27.27\left(\sim 18.94 \mathrm{~cm}^{-1}\right)$ \\
$\mathbf{A}_{\mathbf{2}} / \mathrm{J} \mathrm{mol}^{-1} \mathrm{~K}^{-1}$ & $/$ & $/$ & 9.17 & 38.67 \\
$\boldsymbol{\Theta}_{\boldsymbol{E} 2} / \mathbf{K}$ & $/$ & $/$ & 39.81 & $62.56\left(\sim 43.47 \mathrm{~cm}^{-1}\right)$ \\
$\mathbf{A}_{\mathbf{3}} / \mathrm{J} \mathrm{mol}^{-1} \mathrm{~K}^{-1}$ & $/$ & $/$ & $/$ & 90.16 \\
$\boldsymbol{\Theta}_{\boldsymbol{E} \mathbf{3}} / \mathbf{K}$ & $/$ & $/$ & $/$ & $120.61\left(83.61 \mathrm{~cm}^{-1}\right)$ \\
$\boldsymbol{\Theta}_{\boldsymbol{D}} / \mathbf{K}$ & $/$ & $/$ & $/$ & 149.07 \\
$\mathbf{R}^{\mathbf{2}}$ & 0.9019 & 0.9974 & 0.9996 & 0.9998 \\
$\boldsymbol{\chi}^{\mathbf{2}}$ & 2.31217 & 0.00107 & 0.00016 & 0.00009 \\
\hline
\end{tabular}

Table S9. Detailed calculated parameter in $\mathrm{CsCu}_{4} \mathrm{Se}_{3}$.

\begin{tabular}{cccc}
\hline Parameters & $\boldsymbol{\Gamma}-\mathbf{X}(\boldsymbol{a}$ axis $)$ & $\mathbf{M}-\boldsymbol{\Gamma}(\boldsymbol{b}$ axis $)$ & $\boldsymbol{\Gamma}-\mathbf{Z}(\boldsymbol{c}$ axis $)$ \\
\hline $\boldsymbol{v}_{\mathbf{T A}}(\mathbf{m} / \mathbf{s})$ & 1170 & 1437 & 1316 \\
$\boldsymbol{v}_{\mathbf{T A}}(\mathbf{m} / \mathbf{s})$ & 1414 & 1723 & 1316 \\
$\boldsymbol{v}_{\mathbf{L A}}(\mathbf{m} / \mathbf{s})$ & 2889 & 2777 & 2513 \\
\hline
\end{tabular}

\section{Reference:}

[S1] Kohn, W. Nobel Lecture: Electronic Structure of Matter-Wave Functions and Density Functionals. Reviews of Modern Physics 1999, 71, 1253-1266.

[S2] Kresse, G.; Furthmüller, J. Efficient Iterative Schemes for $a b$ initio Total-Energy Calculations Using A PlaneWave Basis Set. Phys. Rev. B. 1996, 54, 11169-11186.

[S3] Kresse, G.; Joubert, D. From Ultrasoft Pseudopotentials to the Projector Augmented-Wave Method. Phys. Rev. B. 1999, 59, 1758-1775.

[S4] Wang, Y.; Perdew, J. P. Accurate and Simple Analytic Representation of the Electron-Gas Correlation Energy. Phys. Rev. B 1992, 45, 13244-13249. 
[S5] Kresse, G.; Joubert, D. From Ultrasoft Pseudopotentials to the Projector Augmented-Wave Method. Phys. Rev. B 1999, 59, 1758-1775.[S6] Hubbard J. Electron Correlations in Narrow Energy Bands. 1963, 238-257.

[S7] Anisimov, V.; Zaanen, J.; Andersen, O. K. Band theory and Mott Insulators: Hubbard $U$ Instead of Stoner $I$. Phys. Rev. B 1991, 44, 943-954.

[S8] Cococcioni, M.; Gironcoli, S. Linear Response Approach to the Calculation of the Effective Interaction Parameters in the LDA+U Method. Physical Review B. 2005, 71, 035105.

[S9] Dronskowski, R.; Blochl, P. E. Crystal Orbital Hamilton Populations (COHP). Energy-Resolved Visualization of Chemical Bonding in Solids Based on Density-Functional Calculations. J. Phys. Chem. 1993, 97, 8617-8624.

[S10] Maintz, S.; Deringer, V. L.; Tchougreeff, A. L.; Dronskowski, R. Analytic Projection From Plane-Wave and PAW Wavefunctions and Application to Chemical-Bonding Analysis in Solids. J Comput Chem. 2013, 34, 2557-2567.

[S11] Nelson, R.; Ertural, C.; George, J.; Deringer, V. L.; Hautier, G.; Dronskowski, R. LOBSTER: Local Orbital Projections, Atomic Charges, and Chemical-Bonding Analysis From Projector-Augmented-Wave-Based DensityFunctional Theory. J Comput Chem. 2020, 41, 1931-1940.

[S12] Togo, A.; Tanaka, I. First Principles Phonon Calculations in Materials Science. Scripta Materialia 2015, 108, $1-5$.

[S13] Kurosaki, K.; Kosuga, A.; Muta, H.; Uno, M.; Yamanaka, S. Ag TITe $_{5}$ : A High-Performance Thermoelectric Bulk Material with Extremely Low Thermal Conductivity. Appl. Phys. Lett. 2005, 87, 061919.

[S14] Wan, C. L.; Pan, W.; Xu, Q.; Qin, Y. X.; Wang, J. D.; Qu, Z. X. Fang, M. H. Effect of Point Defects on the Thermal Transport Properties of $\left(\operatorname{La}_{\mathrm{x}} \mathrm{Gd}_{1-\mathrm{x}}\right)_{2} \mathrm{Zr}_{2} \mathrm{O}_{7}$ : Experiment and Theoretical Model. Phys. Rev. B 2006, 74, 144109 .

[S15] Tan, G.; Zhao, L. D.; Kanatzidis, M. G. Rationally Designing High-Performance Bulk Thermoelectric Materials. Chem. Rev. 2016, 116, 12123-12149.

[S16] Cahill DG, Watson SK, Pohl RO. Lower limit to the thermal conductivity of disordered crystals. Phys Rev B, 1992, 46: 6131-6140.

[S17] Xie, H. Y.; Su, X. L.; Hao, S. Q.; Zhang, C.; Zhang, Z. K.; Liu, W.; Yan, Y. G.; Wolverton, C.; Tang, X. F.; Kanatzidis, M. G. Large Thermal Conductivity Drops in the Diamondoid Lattice of $\mathrm{CuFeS}_{2}$ by Discordant Atom Doping. J. Am. Chem. Soc. 2019, 141, 18900-18909.

[S18] Xie, H. Y.; Su, X. L.; Zhang, X. M.; Hao, S. Q.; Bailey, T. P.; Stoumpos, C. C.; Douvalis, A. P.; Hu, X. B.; Wolverton, C.; Dravid, V. P.; Uher, C.; Tang, X. F.; Kanatzidis, M. G. Origin of Intrinsically Low Thermal Conductivity in Talnakhite $\mathrm{Cu}_{17.6} \mathrm{Fe}_{17.6} \mathrm{~S}_{32}$ Thermoelectric Material: Correlations between Lattice Dynamics and Thermal Transport. J. Am. Chem. Soc. 2019, 141, 10905-10914.

[S19] Ma, N.; Li, Y. Y.; Chen, L.; Wu, L. M. $\alpha-\mathrm{CsCu}_{5} \mathrm{Se}_{3}$ : Discovery of A Low-cost Bulk Selenide with High Thermoelectric Performance. J. Am. Chem. Soc. 2020, 142, 5293-5303.

[S20] Peplinski, Z.; Brown, D. B.; Watt, T.; Hatfield, W. E.; Day, P. Electrical Properties of $\mathrm{Na}_{3} \mathrm{Cu}_{4} \mathrm{~S}_{4}$, a MixedValence One-Dimensional Metal. Inorg. Chem. 1982, 21, 1752-1755.

[S21] Lin, H.; Tan, G. J.; Shen, J. N.; Hao, S. Q.; Wu, L. M.; Calta, N.; Malliakas, C.; Wang, S.; Uher, C.; Wolverton, C.; Kanatzidis, M. G. Concerted Rattling in $\mathrm{CsAg}_{5} \mathrm{Te}_{3}$ Leading to Ultralow Thermal Conductivity and High Thermoelectric Performance. Angew. Chem. Int. Ed. 2016, 55, 11431-11436.

[S22] Sturza, M.; Bugaris, D. E.; Malliakas, C. D.; Han, F.; Chung, D. Y.; Kanatzidis, M. G. Mixed-Valent $\mathrm{NaCu}_{4} \mathrm{Se}_{3}$ : A Two-Dimensional Metal. Inorg. Chem. 2016, 55, 4884-4890.

[S23] Sturza, M.; Malliakas, C. D.; Bugaris, D. E.; Han, F.; Chung, D. Y.; Kanatzidis, M. G. NaCu $\mathrm{Se}_{4}$ : A Layered Compound with Mixed Valency and Metallic Properties. Inorg. Chem. 2014, 53, 12191-12198.

[S24] Chen, H. J.; Rodrigues, J. B.; Rettie, A. J. E.; Song, T. B.; Chica, D. G.; Su, X. L.; Bao, J. K.; Chung, D. Y.; 
Kwok, W. K.; Wagner, L. K.; Kanatzidis, M .G. High Hole Mobility and Nonsaturating Giant Magnetoresistance in the New 2D Metal $\mathrm{NaCu}_{4} \mathrm{Se}_{4}$ Synthesized by a Unique Pathway. J. Am. Chem. Soc. 2019, 141, 635-642.

[S25] Ma, N.; Jia, F.; Xiong, L.; Chen, L.; Li, Y. Y.; Wu, L. M. $\mathrm{CsCu}_{5} \mathrm{~S}_{3}$ : Promising Thermoelectric Material with Enhanced Phase Transition Temperature. Inorg. Chem. 2019, 58, 1371-1376.

[S26] Huai, W. J.; Shen, J. N.; Lin, H.; Chen, L.; Wu, L. M. Electron-Deficient Telluride $\mathrm{Cs}_{3} \mathrm{Cu}_{20} \mathrm{Te}_{13}$ with SodaliteType Network: Syntheses, Structures, and Physical Properties. Inorg. Chem. 2014, 53, 5575-5580.

[S27] Kim, H. S.; Gibbs, Z. M.; Tang, Y. L.; Wang, H.; Snyder, G. J. Characterization of Lorenz number with Seebeck coefficient measurement. APL Materials 2015, 3, 041506. 